\title{
Dense Wide-Baseline Scene Flow From Two Handheld Video Cameras
}

\author{
Christian Richardt ${ }^{1,2,3} \quad$ Hyeongwoo Kim $^{1} \quad$ Levi Valgaerts $^{1} \quad$ Christian Theobalt $^{1}$ \\ ${ }^{1}$ Max Planck Institute for Informatics $\quad{ }^{2}$ Intel Visual Computing Institute ${ }^{3}$ University of Bath
}

\begin{abstract}
We propose a new technique for computing dense scene flow from two handheld videos with wide camera baselines and different photometric properties due to different sensors or camera settings like exposure and white balance. Our technique innovates in two ways over existing methods: (1) it supports independently moving cameras, and (2) it computes dense scene flow for wide-baseline scenarios. We achieve this by combining state-of-the-art wide-baseline correspondence finding with a variational scene flow formulation. First, we compute dense, wide-baseline correspondences using DAISY descriptors for matching between cameras and over time. We then detect and replace occluded pixels in the correspondence fields using a novel edge-preserving Laplacian correspondence completion technique. We finally refine the computed correspondence fields in a variational scene flow formulation. We show dense scene flow results computed from challenging datasets with independently moving, handheld cameras of varying camera settings.
\end{abstract}

\section{Introduction}

A variety of methods to reconstruct space-time coherent geometry of dynamic scenes from multiple video or depth cameras have been proposed, such as performance capture methods in vision and graphics [29]. Many video-based methods rely on a combination of correspondence finding between camera views to capture shape, and temporal correspondence finding to establish temporal coherence in reconstructions. A deforming template model is often used to assist shape and motion estimation. Most existing approaches are limited to controlled indoor environments with rather dense static camera setups and controlled backgrounds, or are limited to reconstruction of a few dynamic foreground objects, and thus cannot capture the entire scene [34]. In recent years, the trend for dynamic scene reconstruction has been towards increasingly unconstrained capturing of scenes from multiple videos. For instance, first template-based methods to reconstruct humans or faces from stereo cameras with a fixed baseline and in less controlled surroundings, including outdoor scenes, were proposed $[49,56]$.
The widespread proliferation of mobile video cameras, particularly in mobile phones, has accelerated this trend and led to an explosion of recorded video content, which could be used for dynamic scene reconstruction and free-viewpoint rendering, for instance of music or sports events filmed by several spectators [2, 28]. However, videos recorded with mobile cameras pose a new, generalised wide-baseline stereo problem [32]. Correspondences have to be robust to both independent camera motion with wide geometric baselines, as well as starkly differing image characteristics due to different sensors and camera settings, such as exposure and white balance (aka the 'WGSBS' problem [32]). Most existing techniques assume static camera setups, and only few handle moving stereo rigs with fixed baselines, even though videos captured with independently moving handheld cameras of potentially different type are now the norm in practice. In addition to handling these independent cameras, approaches shall also be able to reconstruct dense geometry in both space and time, i.e. shape and motion of all objects in the scene, not only foreground objects for which a template needs to be created in a complex pre-processing step. They also need to succeed with wide-baseline recordings and footage recorded under other adverse effects such as changing lighting and frequent occlusions. Empowering dynamic scene reconstruction methods to handle such scenes requires algorithmic innovation on several ends. In this paper, we take a step towards this goal by proposing a new solution to dense correspondence finding in more general settings.

Most dynamic scene reconstructions are based on the estimation of the 3D scene motion over time, which is known as scene flow, a term coined by Vedula et al. [50] in analogy to the term 'optical flow' for the motion over time. Our goal is to compute dense scene flow of general dynamic scenes from two handheld videos of independently moving cameras with wide baseline in terms of both camera geometry and sensor characteristics. This is not supported by current scene flow techniques: dense approaches generally rely on narrow camera baselines [5, 21, 48], and wide-baseline approaches are not dense as they use sparse scene representations such as voxels or particles $[10,15,50]$. In addition, previous techniques do not support different sensors and handheld videos, as they assume constant camera calibration over time. 
We propose a technique that overcomes these limitations by combining wide-baseline correspondence finding with a dense, variational scene flow computation approach that jointly estimates dense correspondence fields across camera views and within camera views of two subsequent time steps, even if sensor or image characteristics differ notably between cameras. Our technical contributions are (1) a novel correspondence finding technique that uses DAISY descriptors [45] for wide-baseline matching in both space (between cameras) and time (in the same camera view), and is optimised using PatchMatch belief propagation (РMBP) [6], and (2) an edge-preserving Laplacian correspondence completion technique. We show dense scene flow results, alongside with dense stereo geometry, computed from challenging independently moving handheld video datasets with medium to wide camera baselines, which we will make publicly available.

\section{Related work}

Scene flow describes the motion within a scene over time, specifically the motion of every visible 3D point between two time steps. Many techniques have been proposed to compute the scene flow from two or more videos, including voxel coloring from dense in-studio camera setups [50], surfel tracking [10], and growing correspondence seeds [8]. Scene flow was also computed as part of non-rigid scene registration [4], and by means of particle-based estimation [15]. However, the most common class of scene flow approaches are variational methods [5, 12, 21, 23, 36, 43, 44, 48, 53], which provide dense, continuous and strongly regularised solutions. Some techniques enforce motion priors such as affine [60] or piecewise rigid motions [23, 30, 52], but these are violated by the non-rigid scenes we are targeting with our approach. Many recent techniques also build on RGB-D data obtained from consumer depth sensors [12, 15, 19, 20, 23, 26, 37, 43, 58]. However, these approaches are limited to indoor use due to the depth sensors, while we target general, unconstrained outdoor settings with normal video cameras. Like most techniques, we compute scene flow between exactly two time steps; only few techniques enforce temporal consistency over multiple time steps [22, 51]. We also assume synchronised input videos, which can be achieved in hardware or software [11, 14, 18, 31].

Even though some dense variational approaches are able to handle moving cameras $[48,53]$, they assume a static camera rig with a fairly narrow baseline $(10-30 \mathrm{~cm})$ and fail with wider camera baselines or when cameras are moving independently, as in our case. On the other hand, methods that succeed on wider baselines only reconstruct sparse correspondences. In contrast, our method captures dense scene flow and stereo geometry also with wider baselines and independently moving cameras.

Scene flow estimation is also related to non-rigid structure from motion $[1,9,13,16,35,41,57,61]$, but these approaches make strong prior assumptions about scene motion models and work best with small displacements between video frames. Our work is also related to spatio-temporal stereo matching, which has been demonstrated for static camera setups and controlled scenes [24, 39, 59]. As stated in the introduction, scene flow is an important ingredient for many applications, including 3D motion understanding [30, 53], facial performance capture $[49,56]$ and free-viewpoint video [28]. This paper paves the way to lifting these applications to the case of independent handheld video in general scenes.

Wide-baseline matching addresses the difficult task of finding corresponding points in potentially very different viewpoints. The most robust matching results have been achieved using affinely invariant features [33, 47]. While robust, these techniques are very sparse as they only produce a few hundred correspondences per image pair. Follow-up work hence explored densification using a multi-resolution variational formulation [42] or match propagation [25]. To avoid a separate densification step, the DAISY descriptor [45] we use was specifically designed for dense wide-baseline matching [46]. We thus use insights from the design of DAISY and adapt it to the case of dense matching across camera views and over time.

\section{Method}

Our approach computes a dense reconstruction of geometry and scene flow from dynamic scenes casually captured with two handheld video cameras, without imposing any specific assumptions about the scene structure or camera motion. Cameras can differ in make and sensor characteristics, and our method is one of the first to tolerate notable appearance differences between videos.

We distinguish three kinds of correspondences, which we all call flows for simplicity: stereo flow is the correspondence between images from different cameras at the same time, optical flow is correspondence over time within a camera, and scene flow describes the 3D motion over time. We use a pipeline with four main stages:

1. synchronisation and calibration,

2. correspondence finding,

3. occlusion filling, and

4. scene flow computation.

We first synchronise the input videos and calibrate the cameras, before we estimate bidirectional correspondences between pairs of images using the same novel technique for both stereo flow (between cameras at the same time) and optical flow (same camera over time). The stereo correspondences are then postprocessed by invalidating and filling occlusions using a novel edge-preserving scheme based on local linear regression. We finally refine the computed correspondence fields in a variational scene flow formulation. 


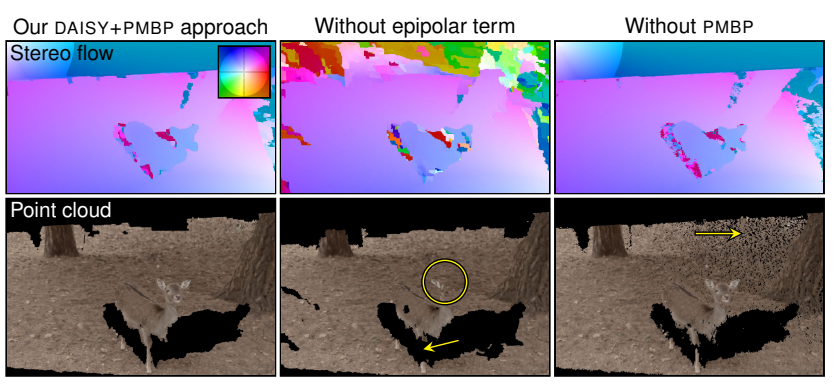

Figure 1. Our DAISY+PMBP correspondences compared to leaving out the epipolar term $\left(w_{\mathrm{E}}=0\right)$ or PMBP $\left(w_{\mathrm{p}}=0\right)$, which results in missing parts like the deer's head (centre) and incoherent geometry (right), respectively.

Calibration We assume that our input videos have known, fixed camera intrinsics, and are synchronised temporally, which can be done automatically by video-based methods $[11,14]$. Similar to most approaches looking into wide-baseline stereo reconstruction, we calibrate the moving cameras extrinsically by undistorting all input video frames and then estimating the camera geometry using structure-from-motion techniques with fixed intrinsics [55, 63].

\subsection{Correspondence finding with DAISY + PMBP}

Our correspondence finding strategy is based on the DAISY descriptor by Tola et al. [45]. In contrast to most other descriptors, such as SIFT or SURF, which were designed for describing sparse interest points [33, 47], DAISY was designed for finding dense correspondences, specifically in wide-baseline scenarios. The DAISY descriptor encodes local appearance using image gradient histograms across different gradient orientations. The gradients are computed at different image scales for different points around the descriptor location, depending on their distance to it. This results in a flower-like arrangement - hence the name DAISY. However, pixel-wise local matching can give rise to spatial inconsistencies. We propose a matching scheme that establishes geometric and spatial coherence by introducing an epipolar energy term and a global matching scheme (see Figure 1).

We use DAISY as the key ingredient in our matching cost $c(\mathbf{x}, \mathbf{y})$ between image locations $\mathbf{x}$ and $\mathbf{y}$ in different images:

$$
c(\mathbf{x}, \mathbf{y})=c_{\mathrm{D}}(\mathbf{x}, \mathbf{y})+c_{\mathrm{C}}(\mathbf{x}, \mathbf{y})+c_{\mathrm{E}}(\mathbf{x}, \mathbf{y}),
$$

which combines the DAISY descriptor difference $c_{\mathrm{D}}$ with a colour consistency term $c_{\mathrm{C}}$, and an epipolar term $c_{\mathrm{E}}$ (only used for stereo flows). We next describe each of these terms, and then discuss how we minimise the matching costs across all pixels to compute correspondence fields.

The DAISY term measures the dissimilarity of local image regions using the difference between the DAISY descriptors $\mathbf{D}(I, \mathbf{x})$ computed at the two considered locations $\mathbf{x}$ and $\mathbf{y}$ in images $I_{1}$ and $I_{2}$ :

$$
c_{\mathrm{D}}(\mathbf{x}, \mathbf{y})=w_{\mathrm{D}} \cdot\left\|\mathbf{D}\left(I_{1}, \mathbf{x}\right)-\mathbf{D}\left(I_{2}, \mathbf{y}\right)\right\|_{2}^{2},
$$

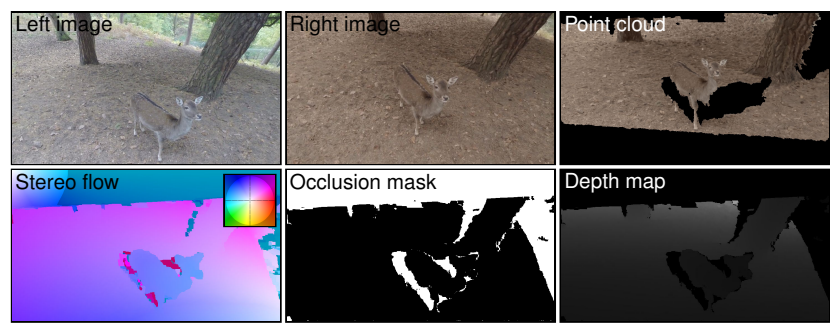

Figure 2. Two input frames from our handheld DEER dataset with different exposure and white balance, which are captured about $65 \mathrm{~cm}$ apart with an angle of about 13 degrees between the optical axes, and the resulting triangulated point cloud (top right). Below: estimated stereo correspondences, occlusion mask and depth map.

where $w_{\mathrm{D}}$ is the weight for this term. As suggested by the authors of DAISY, we orient descriptors along the epipolar lines when epipolar geometry is given. For example, the descriptor at $\mathbf{x}$ is oriented along the epipolar line $\mathbf{l}=\mathbf{F}^{\top} \mathbf{y}$. We use the authors' implementation of the descriptor (aka 'libdaisy'), but to preserve more geometric details, we use a smaller footprint of only two rings with a radius of 10 pixels for stereo flow computation.

The colour consistency term $c_{\mathrm{C}}$ helps to disambiguate image regions with similar gradient distributions, for example in areas of constant colour. In those regions, DAISY descriptors are often similar, but the colour term penalises different colours that would result in mismatches. We compute the colour term using

$$
c_{\mathrm{C}}(\mathbf{x}, \mathbf{y})=w_{\mathrm{C}} \cdot\left\|\mathbf{A} I_{1}(\mathbf{x})+\mathbf{a}-I_{2}(\mathbf{y})\right\|_{2},
$$

where $w_{\mathrm{C}}$ is the colour term weight, and the $3 \times 3$ matrix A and offset a apply an affine colour transformation that adjusts the colours in image $I_{1}$ to be closer to those in image $I_{2}$ (in RGB colour space). This compensates for differences in exposure and white balance between the images (e.g. see Figure 2). At first, we initialise $\mathbf{A}$ to the identity matrix and a to the zero vector, but in later iterations, we estimate them with a least-squares fit to the colours of corresponding pixels.

The epipolar term $c_{\mathrm{E}}$ measures how well two image points $\mathrm{x}$ and $\mathbf{y}$ satisfy the epipolar geometry defined by the fundamental matrix $\mathbf{F}$. In stereo correspondence, this term helps to constrain correspondences to lie close to each others' epipolar lines. This reduces the search space and mismatches. We use the Sampson distance as described by Hartley and Zisserman [17, Section 11.4.3]:

$$
c_{\mathrm{E}}(\mathbf{x}, \mathbf{y})=\frac{w_{\mathrm{E}} \cdot\left(\mathbf{y}^{\top} \mathbf{F} \mathbf{x}\right)^{2}}{(\mathbf{F} \mathbf{x})_{1}^{2}+(\mathbf{F x})_{2}^{2}+\left(\mathbf{F}^{\top} \mathbf{y}\right)_{1}^{2}+\left(\mathbf{F}^{\top} \mathbf{y}\right)_{2}^{2}},
$$

where $w_{\mathrm{E}}$ is the weight for the epipolar term, and $(\mathbf{F x})_{k}^{2}$ represents the square of the $k$-th entry of the vector $\mathbf{F x}$.

We want to minimise the matching cost in Equation 1 across all pixels in a locally smooth way. For this, we chose a variant of PatchMatch [3], as its stochastic initialisation pro- 
vides good initial correspondences even in challenging widebaseline cases. Smoothness of the correspondence field is encouraged using the PatchMatch belief propagation (РMBP) technique by Besse et al. [6], which introduces a pairwise term $p$ for regularisation into the energy formulation:

$$
E=\underbrace{\sum_{i} c\left(\mathbf{x}_{i}, \mathbf{y}_{i}\right)}_{\text {unary terms }}+\underbrace{\sum_{i} \sum_{j \in N(i)} p\left(\mathbf{x}_{i}, \mathbf{y}_{i}, \mathbf{x}_{j}, \mathbf{y}_{j}\right)}_{\text {pairwise terms }},
$$

where $N(i)$ represents the set of 4-neighbours of pixel $i$. We use the truncated squared difference between flows as our pairwise term to enforce smoothness:

$$
p\left(\mathbf{x}_{1}, \mathbf{y}_{1}, \mathbf{x}_{2}, \mathbf{y}_{2}\right)=\min \left(\tau_{\mathrm{p}}, w_{\mathrm{p}} \cdot\left\|\left(\mathbf{y}_{1}-\mathbf{x}_{1}\right)-\left(\mathbf{y}_{2}-\mathbf{x}_{2}\right)\right\|_{2}^{2}\right),
$$

using the threshold $\tau_{\mathrm{p}}$ and weight $w_{\mathrm{p}}$. We use PMBP to compute bidirectional correspondences between each pair of images, so that we can easily check for their consistency in subsequent computation steps.

We refine our correspondences over multiple passes in which we estimate the affine colour transform $[\mathbf{A} \mathbf{a}]$ after each run of PMBP. We use the same settings for all sequences, which illustrates the stability of our approach across differing camera responses. We keep the parameters $\left(w_{\mathrm{D}}, \tau_{\mathrm{p}}\right)=(1,50)$ constant for all passes, but vary the other parameters. For stereo correspondence, we use four passes, each with two iterations of $\mathrm{PMBP}$. In the first pass, we use equal weights $w_{\mathrm{D}}=w_{\mathrm{C}}=w_{\mathrm{E}}=1$, but set the colour weight $w_{\mathrm{C}}$ to 10 after estimating the colour transform in the first pass. We also increase the pairwise weight $w_{\mathrm{p}}$ across passes (using values $0.01,0.02,0.1,1)$ to more strongly enforce smoothness as matching progresses. This produces results such as shown in Figure 2. For optical flow, we use only two passes, with 6 and 4 PMBP iterations respectively, with parameters $\left(w_{\mathrm{D}}, w_{\mathrm{C}}, w_{\mathrm{E}}\right.$, $\left.w_{\mathrm{p}}\right)=(1,20,0,0.01)$. In the first pass, and for optical flow only, we speed up computation using precomputed DAISY descriptors for all pixels.

\subsection{Laplacian occlusion filling}

Pairwise correspondences are incorrect in areas of occlusion, where a point is only visible in one view, but not the other. In these cases, the correspondences computed in the previous section are mismatches, which would negatively impact the variational scene flow estimation in the next section. We therefore invalidate and fill in occluded pixels in the stereo flows. As wide-baseline views can cause large occlusion regions, simple occlusion filling strategies, such as diffusion or weighted median filtering, cannot handle them adequately. We propose a new occlusion filling method based on the observation that flow values are linearly correlated with colour intensities within a small window, which we exploit using local linear regression. This gives more cues for the occlusion filling than just using the surface. Figure 3 illustrates this:

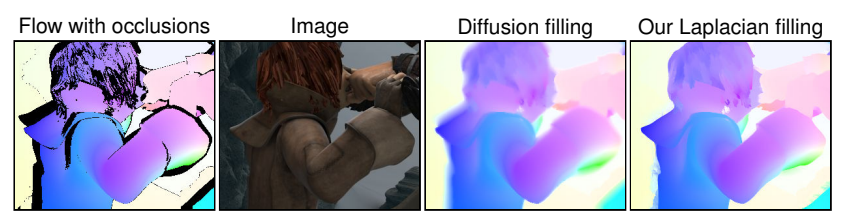

Figure 3. Our Laplacian occlusion filling preserves images edges in the filled flow fields better than a simple diffusion fill. Data from MPI-Sintel [7].

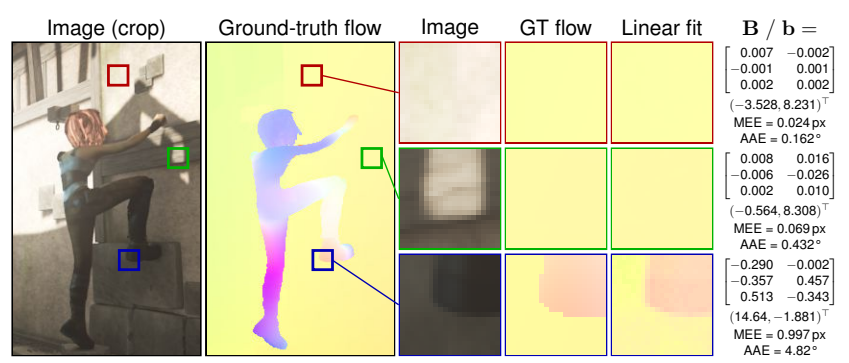

Figure 4. Visualisation of the local linearity between 8-bit RGB colours in a $21 \times 21$ window and the corresponding flow values (Equation 7) for three examples: constant colour and flow (red, top), constant flow despite textured patch (green, middle), and image edges coinciding with flow discontinuities (blue, bottom). Error measures are mean endpoint error (MEE) and average angular error (AAE). Data from MPI-Sintel [7].

most edges are sharp in our filled flow field. However, note that the flow is imperfect on the girl's back due to the lack of strong image edges in this area. In such cases, our filling result degrades gracefully, and still is better than diffusion.

We begin by computing a binary occlusion mask from our bidirectional stereo flows using the forward-backward check with a threshold of 3 pixels. This mask, which can optionally be cleaned using morphological closing, determines which pixels are invalidated and will be filled in in the next step.

The key assumption is that the flow $\mathbf{u}_{i}=\mathbf{y}_{i}-\mathbf{x}_{i}$ between corresponding points $\mathbf{x}_{i}$ and $\mathbf{y}_{i}$ can be expressed as a linear function of the image colours within a small window $w$ (in practice $3 \times 3$ pixels):

$$
\mathbf{u}_{i} \approx \mathbf{B} I\left(\mathbf{x}_{i}\right)+\mathbf{b}, \quad \text { for all } i \in w,
$$

where $\mathbf{B}$ is a $2 \times 3$ matrix, $I\left(\mathbf{x}_{i}\right)$ a $3 \times 1 \mathrm{RGB}$ vector and b a $2 \times 1$ vector. Figure 4 visualises the good fit of local linearity for three example windows. In practice, we use $3 \times 3$ windows which provide an even better fit than the shown examples. This linear relationship applies to every window a pixel is in, so we sum up all overlapping windows:

$$
E=\sum_{j} \sum_{i \in w_{j}}\left\|\mathbf{u}_{i}-\left(\mathbf{B}_{j} I\left(\mathbf{x}_{i}\right)+\mathbf{b}_{j}\right)\right\|^{2}+\epsilon \cdot\left\|\mathbf{B}_{j}\right\|_{F}^{2},
$$

where $w_{j}$ is a window of pixels around $j, \epsilon=10^{-4}$, and the regularisation term on $\mathbf{B}_{j}$ is included for numerical stability (as for a constant image, $\mathbf{B}$ and $\mathbf{b}$ cannot be determined uniquely), and for a smoother solution (since $\|\mathbf{B}\|_{F}^{2}=0$ 


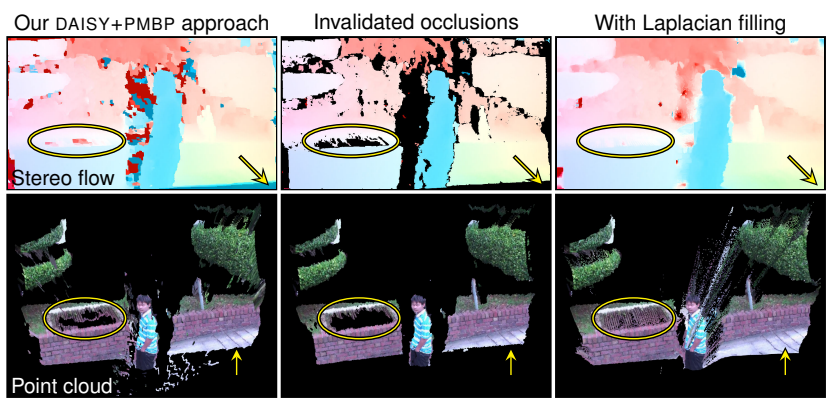

Figure 5. Starting from the DAISY+PMBP flow (left), we invalidate occluded pixels (centre), and fill them plausibly using a novel edgepreserving Laplacian filling technique (right).

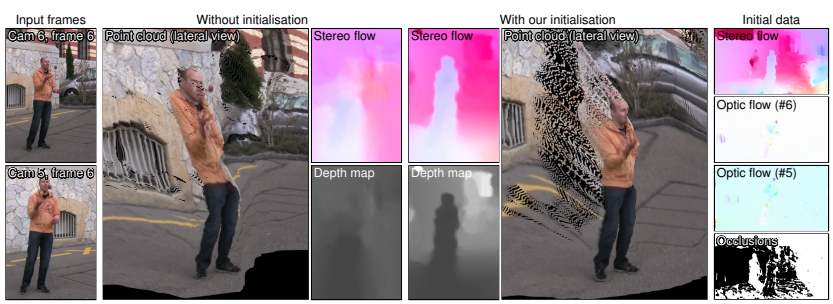

Figure 6. Comparison of scene flow computation without (left) and with (right) initialisation from DAISY+PMBP on the JUGGLER dataset [2] with wide camera baseline. Without outside initialisation, the juggler is not reconstructed correctly as seen clearly in the point cloud, stereo flow and depth map. With our DAISY+PMBP initialisation, using the flows and occlusion mask shown on the far right, the juggler is reconstructed correctly.

implies that $\mathbf{u}$ is constant over the window). Levin et al. [27] provide a closed-form solution for this sort of cost function, by eliminating their equivalent of our $\mathbf{B}$ and $\mathbf{b}$ terms, which results in a quadratic cost in the unknowns $\mathbf{U}$ alone:

$$
E^{\prime}=\mathbf{U}^{\top} \mathbf{L} \mathbf{U}
$$

where $\mathbf{U}$ is an $N \times 2$ matrix with $\mathbf{u}_{i}^{\top}$ as its $i$ th row, and $\mathbf{L}$ is the so-called matting Laplacian [27, Eq. 12].

We augment this cost with constraints and differentiate with respect to $\mathbf{U}$, resulting in the sparse linear system

$$
\left(\mathbf{L}+\lambda \cdot \mathbf{D}_{\mathrm{C}}\right) \mathbf{U}=\lambda \cdot \mathbf{U}_{\mathrm{C}},
$$

where $\mathbf{D}_{\mathrm{C}}$ is a diagonal matrix with one for non-occluded pixels and zero for all other pixels, weighted by $\lambda=5$, and $\mathbf{U}_{\mathrm{C}}$ is a matrix containing the specified correspondences for non-occluded pixels and zero for other pixels. In our implementation, we compute the Cholesky factorisation of $\left(\mathbf{L}+\lambda \cdot \mathbf{D}_{\mathbf{C}}\right)$ once and solve Equation 10 separately for each column of $\mathbf{U}$. Figure 5 shows an example where areas like the ground and the grating on the left are filled with plausible flow values.

\subsection{Scene flow computation}

To compute the scene flow between adjacent time steps, we build upon the variational scene flow method by Valgaerts et al. [48], which we initialise using the flows computed in the previous sections. We take inspiration from EpicFlow [38], which achieved state-of-the-art optical flow results by combining robust dense matching with a variational refinement. This combination has two major advantages for scene flow computation. First, when applied to wide-baseline videos with large parallax between views, the used variational approach fails to converge to the correct solution without a reasonable initialisation, which our flows provide (see Figure 6). Second, variational methods have the key advantage over stochastic optimisation methods like PMBP that their strong regularisation leads to smoother results. In our case, the stereo flows computed with the methods in Sections 3.1 and 3.2 are accurate at the pixel level, but have sub-pixel noise. When triangulating the 3D positions of these flows, the sub-pixel noise manifests itself as noisy 3D positions.

We therefore use quarter-resolution downsampled versions of our stereo and optical flows as initialisation for the variational scene flow computation, which computes smoother and refined flows at full image resolution in a multi-resolution fashion. In short, the method of Valgaerts et al. [48] estimates the scene flow between two successive time steps by minimising an energy functional of the form

$$
E=\int_{\Omega}(\underbrace{\sum_{i=1}^{4} E_{\mathrm{D}}^{i}}_{\text {data }}+\underbrace{\sum_{i=1}^{2} \alpha_{i} \cdot E_{\mathrm{E}}^{i}}_{\text {epipolar }}+\underbrace{\sum_{i=1}^{3} \beta_{i} \cdot E_{\mathrm{S}}^{i}}_{\text {smoothness }}) \mathrm{d} \mathbf{x} .
$$

For completeness, we discuss the individual energy terms in Appendix A. We finally obtain the scene flow as the difference between the triangulated 3D positions of temporally corresponding points at time $t$ and $t+1$. We show example results in Figure 7, with two of the four input video frames, the refined optical and stereo flows, and a visualisation of the scene flow on a point-cloud reconstruction using every hundredth scene flow vector.

\section{Results and discussion}

To our knowledge, we propose the first dense scene flow technique for handheld, independently moving cameras with wide baselines in both the geometric and photometric sense. In Figure 7, we show scene flow results from handheld cameras on our DEER and BOAR datasets, and Jiang et al.'s BEAR and BOY datasets [24], where we show two frames covering about a second of time. Notice the different colours and exposure in the DEER and BOAR datasets, and how the cameras move and shake over time. We are nonetheless able to faithfully capture dense stereo and scene flows. The BOY dataset, in particular, shows considerable camera motion, which can be seen in the changing colours of the optical flow visualisation. In Figure 8, we furthermore show results for moving stereo rigs, where the cameras are not moving independently, 
- DEER sequence (200 frames, independent handheld cameras, with white-balance and exposure differences) -
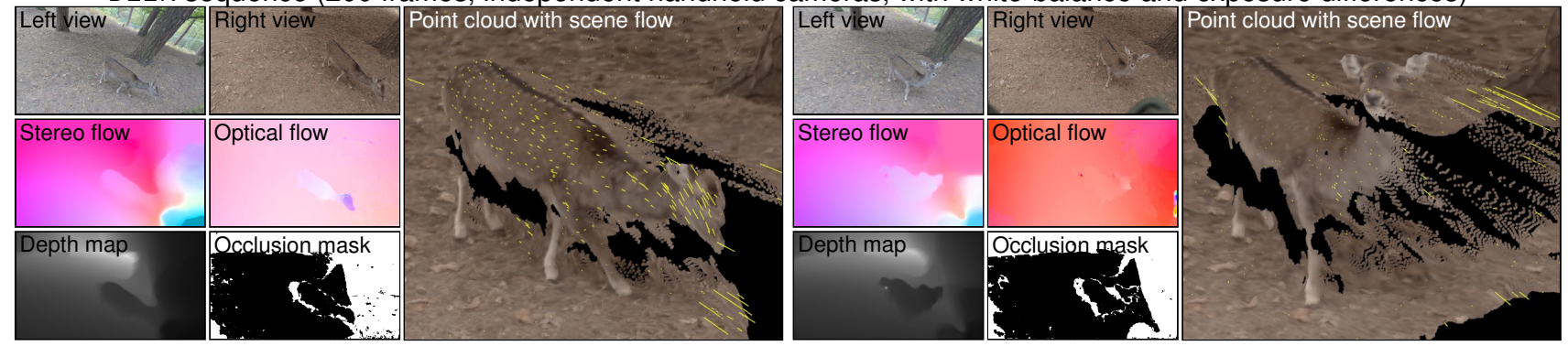

- BOAR sequence (69 frames, independent handheld cameras, with white-balance and exposure differences) -
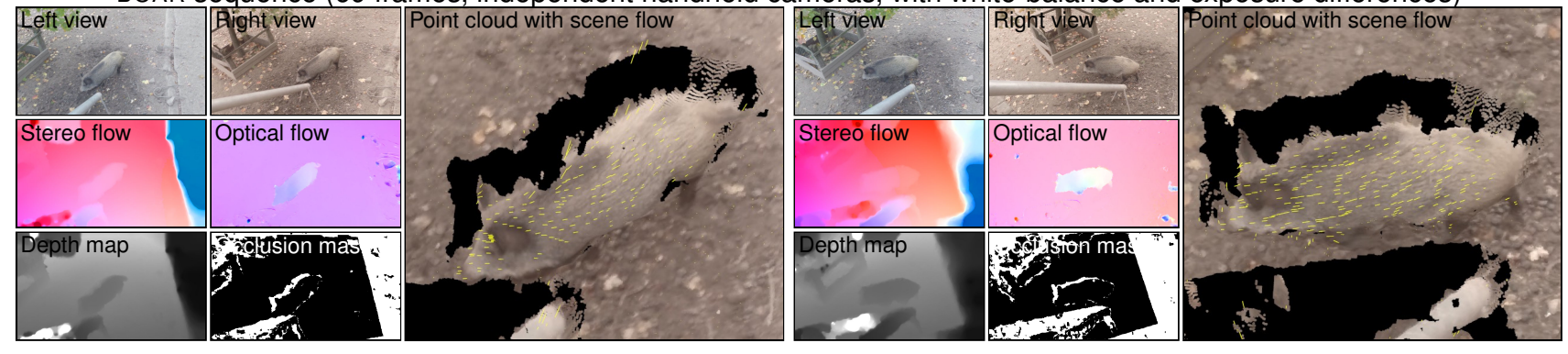

- BEAR sequence [24] (36 frames, independent handheld cameras, similar photometrics) -
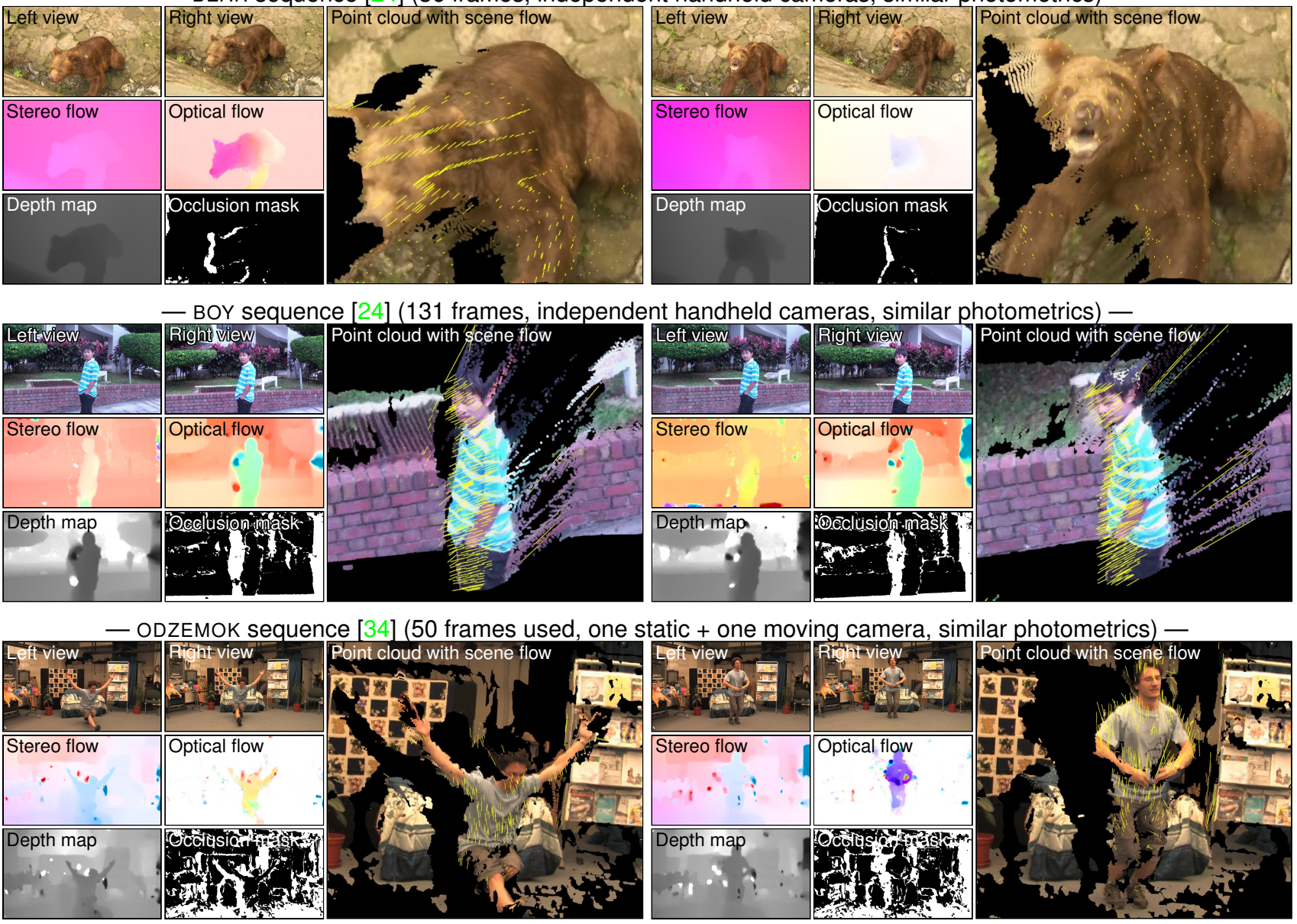

Figure 7. Results of our scene flow technique on the DEER, BOAR, BEAR and BOY datasets (all handheld), and the ODZEMOK dataset. The smaller images show pairs of input frames (top), visualisations for stereo and optical flows (middle; scaled differently for visualisation), and depth map and occlusion mask (bottom; all for left view). The large images show cropped point cloud reconstructions with a subset of the scene flow vectors. Our approach can cope with the considerable differences in the camera and sensor characteristics in the DEER dataset, and also the considerable camera motion and shake, which causes the changing colours of the optical flow visualisations. 


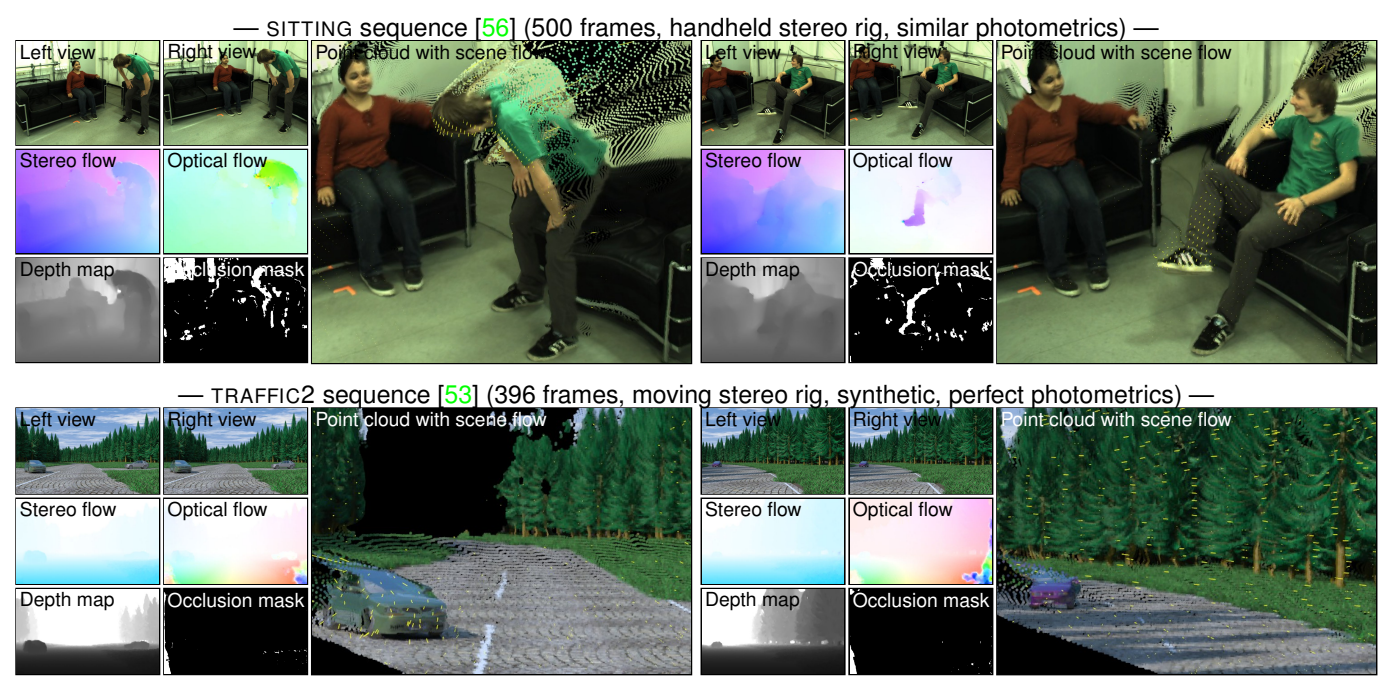

Figure 8. Results of our scene flow technique for moving stereo rigs on the handheld SITTING [56] and synthetic TRAFFIC2 [53] datasets.

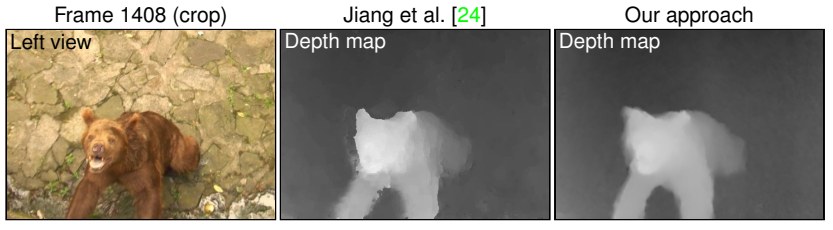

Figure 9. Comparison of depth maps for the BEAR sequence by Jiang et al. [24] and our approach. Our variational refinement produces smoother depth variations on the bear and the background, and only slightly blurrier depth boundaries.

but are fixed in a rig. Figure 9 compares our depth map to one computed by Jiang et al.'s handheld stereo technique [24], and shows that our result has smoother depth variations thanks to our variational scene flow refinement. Please see our supplemental video for video clips of our results.

Most dense two-view scene flow techniques use a narrow camera baseline (tens of $\mathrm{cm}$ ) and fail for wider baselines. We show an example in Figure 6, in which we compare the variational scene flow technique described in Section 3.3 without and with our flow initialisation. Without our flow initialisation, stereo and optical flows are computed in a coarse-to-fine manner over more than 50 pyramid levels. The incremental flow refinement at each pyramid level fails to reconstruct the juggler, whereas our flow initialisation provides the necessary input to reconstruct him correctly.

Occlusion filling We more thoroughly evaluate our Laplacian occlusion filling technique on MPI-Sintel [7]. We invalidate flow pixels marked in the provided occlusion maps, fill them using our technique and compare to the ground-truth flow fields with diffusion-based filling [54] as baseline. For the first 10 frames of the 23 training datasets, Laplacian filling has a mean endpoint error of 0.92 pixels (diffusionbased: 1.34$)$, and an average angular error of $5.7^{\circ}\left(6.4^{\circ}\right)$. Laplacian filling has the smallest mean endpoint error for all
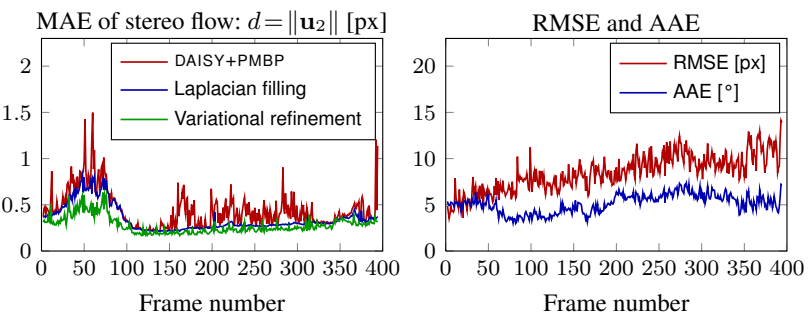

Figure 10. Per-frame error statistics for the TRAFFIC2 dataset [53]. Each step in our pipeline reduces the mean absolute error (MAE) of the estimated stereo flows (left). The right graph shows the total error of our scene flow, in terms of RMSE and AAE.

230 frames. This means that the assumption of correlated colour and flow edges is valid in most cases.

Ground-truth evaluation There are no existing groundtruth datasets for evaluating scene flow estimated from handheld cameras, let alone the wide-baseline case our approach addresses. We therefore quantitatively evaluate our scene flow results using the synthetic dataset TRAFFIC2 [53] with ground-truth flows. This dataset models a car-mounted stereo camera as the car drives along a road. As such, the cameras are fixed in a static rig and do not move independently, but this is the closest available synthetic dataset to our application scenario. In Figure 10, we plot per-frame error statistics across the whole sequence. Using the same notation as Wedel et al. [53], we evaluate the stereo flow using the mean absolute error $\mathrm{MAE}_{d}=\frac{1}{|\Omega|} \sum_{\Omega}|d-\tilde{d}|$, where $\Omega$ is the domain of all image pixels and $d=\left\|\mathbf{u}_{2}\right\|_{2}$ is the estimated ${ }^{1}$ and $\tilde{d}$ the ground-truth disparity. This metric excludes occluded regions. The top of Figure 10 shows that each of our processing steps reduces the error in the estimated stereo flow fields. The scene flow is evaluated using the root mean squared

\footnotetext{
${ }^{1}$ Our approach does not assume rectified stereo images and the stereo flow $\mathbf{u}_{2}$ is therefore not restricted to be horizontal, so we use its $\ell_{2}$ norm.
} 


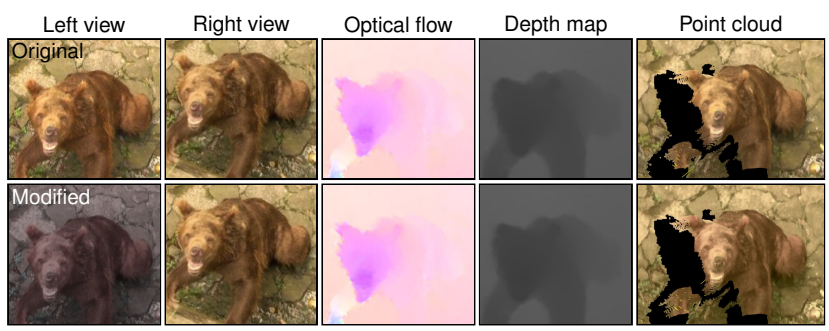

Figure 11. Our approach is robust to photometric differences in the input videos: with modified input colours (bottom), we obtain results comparable to the clean videos (top).

error (RMSE) and the average angular error (AAE):

$$
\begin{aligned}
\operatorname{RMSE} & =\sqrt{\frac{1}{|\Omega|} \sum_{\Omega}\|(u, v, d, p)-(\tilde{u}, \tilde{v}, \tilde{d}, \tilde{p})\|^{2}}, \\
\mathrm{AAE} & =\frac{1}{|\Omega|} \sum_{\Omega} \cos ^{-1} \frac{(u, v, p, 1) \cdot(\tilde{u}, \tilde{v}, \tilde{p}, 1)}{\|u, v, p, 1\| \cdot\|\tilde{u}, \tilde{v}, \tilde{p}, 1\|} .
\end{aligned}
$$

Artificial contamination To demonstrate the robustness of our method to photometric differences, we contaminated one video of the BEAR dataset, and still obtained comparable reconstruction results to the clean input videos (Figure 11).

Runtimes Our correspondence finding takes about six minutes for computing optical flow or stereo flow between an image pair with $960 \times 540$ resolution. The occlusion filling takes 7 seconds, and the variational refinement 1.6 minutes for each set of four frames (single-threaded run times on a $3.5 \mathrm{GHz}$ Xeon CPU).

Extrinsic self-calibration We experimented with estimating epipolar geometry, and hence relative extrinsic calibration, in our DAISY+PMBP approach in Section 3.1, but we found that a global extrinsic calibration of the input videos using structure-from-motion produces more stable results, as all cameras share the same global coordinate system.

Failure cases Like all scene flow techniques, our proposed technique fails if the stereo or optical flows contain incorrectly matched correspondences. This can for example happen if scene motion is too fast, leading to inaccurate optical flow estimation, which impacts the quality of the scene flow estimation. We show such a case for two camera views from the BREAKDANCERS dataset [62] in Figure 12. The main source of mismatches in our optical and stereo flows are large areas of constant colour, which are not sufficiently regularised by $\mathrm{PMBP}$, and incorrect correspondences in occlusion regions that are not detected as such. The resulting spurious scene flow can be seen in Figure 12, on the floor and the background.

Limitations Camera baselines wider than about $30^{\circ}$ prove problematic, but could potentially be handled with affineinvariant descriptors. We also observe that depth discontinuities are somewhat blurred spatially, which results in 'rubber

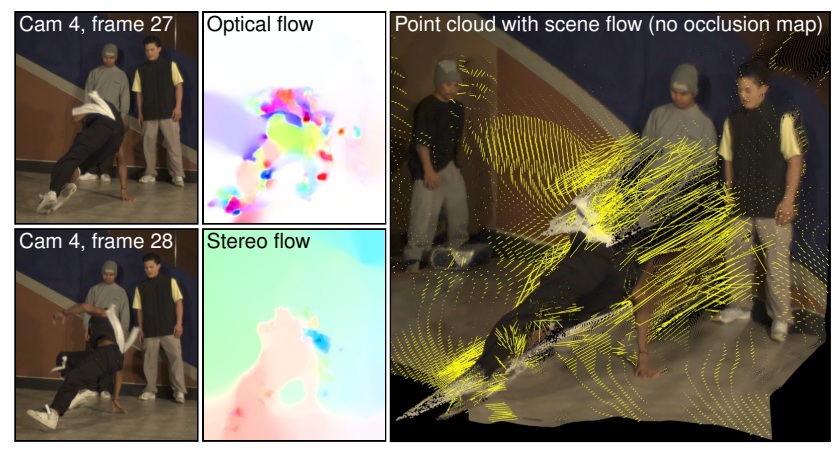

Figure 12. Example of a failure case: the motion between frames of the BREAKDANCERS dataset [62] (using two out of eight cameras) is too fast (see images on the left), leading to poor optical flow estimation and hence poor scene flow.

sheet' artefacts. This could be addressed using edge-aware regularisation.

Discussion We understand our work as a step towards unconstrained dynamic 3D scene reconstruction in general environments and from just a few handheld videos. In practice, most mobile cameras are not calibrated and most videos are not synchronised, so an important direction for the future are algorithms that can jointly estimate calibration and synchronisation parameters, in addition to the dynamic geometry. Perhaps one could also exploit the increased temporal resolution obtained from rolling shutter or when cameras are not frame-synchronised. Real videos are also affected by motion blur, lens flares and changing lens parameters such as zoom and aperture, which all have a negative impact on correspondence finding, but could also be exploited to gain additional information about the scene.

\section{Conclusion}

We presented a dense scene flow technique for two general handheld videos, captured with wide camera baseline, and different camera and sensor characteristics. Our technique supports wider baselines than previous dense scene flow techniques by virtue of a novel wide-baseline correspondence finding approach built on DAISY descriptors and colour consistency adjustment with PMBP optimisation. We improve stereo and optical flows computed in the process of scene flow estimation using a new edge-aware Laplacian occlusion filling method that exploits image information to complete previously invalided occluded pixels. We finally refine all flows in a variational scene flow formulation, to obtain dense, smooth correspondences across space and time. This combination of techniques enables dense scene flow and stereo geometry computation from handheld videos, which we demonstrated on a range of challenging datasets with complex motions.

Acknowledgements We thank the authors of the used datasets. Funded by ERC Starting Grant 335545 CapReal. 


\section{A. Variational scene flow computation}

The method of Valgaerts et al. [48] estimates the scene flow between two successive time steps by minimising an energy functional of the form

$$
E=\int_{\Omega}(\underbrace{\sum_{i=1}^{4} E_{\mathrm{D}}^{i}}_{\text {data }}+\underbrace{\sum_{i=1}^{2} \alpha_{i} \cdot E_{\mathrm{E}}^{i}}_{\text {epipolar }}+\underbrace{\sum_{i=1}^{3} \beta_{i} \cdot E_{\mathrm{S}}^{i}}_{\text {smoothness }}) \mathrm{d} \mathbf{x} .
$$

The first part of this energy collects four data terms that measure the difference in brightness between corresponding points in the four-frame configuration of Figure 13:

$$
\begin{aligned}
& E_{\mathrm{D}}^{1}=\Psi\left(\left\|I_{1}^{t+1}\left(\mathbf{x}+\mathbf{u}_{1}\right)-I_{1}^{t}(\mathbf{x})\right\|_{2}^{2}\right) \\
& E_{\mathrm{D}}^{2}=\Psi\left(\left\|I_{2}^{t+1}\left(\mathbf{x}+\mathbf{u}_{1}+\mathbf{u}_{2}+\mathbf{u}_{3}\right)-I_{2}^{t}\left(\mathbf{x}+\mathbf{u}_{2}\right)\right\|_{2}^{2}\right), \\
& E_{\mathrm{D}}^{3}=\Psi\left(\left\|I_{2}^{t}\left(\mathbf{x}+\mathbf{u}_{2}\right)-I_{1}^{t}(\mathbf{x})\right\|_{2}^{2}\right), \\
& E_{\mathrm{D}}^{4}=\Psi\left(\left\|I_{2}^{t+1}\left(\mathbf{x}+\mathbf{u}_{1}+\mathbf{u}_{2}+\mathbf{u}_{3}\right)-I_{1}^{t+1}\left(\mathbf{x}+\mathbf{u}_{1}\right)\right\|_{2}^{2}\right) .
\end{aligned}
$$

Here, $\mathbf{u}_{1}$ and $\mathbf{u}_{2}$ denote the optical flow in the first view and the stereo flow at time $t$, respectively, while $\mathbf{u}_{3}$ closes the correspondence loop from $I_{1}^{t}$ to $I_{2}^{t+1}$. The images $I_{1}^{t}$ are colour-corrected to match $I_{2}^{t}$ using the transform $\left[\begin{array}{ll}\mathbf{A} & \mathbf{a}\end{array}\right]$ estimated for Equation 3 - without this appearance normalisation, matching would be much harder. To handle the remaining appearance differences, we also include the gradient difference for improved matching in the presence of noise and lighting changes over time. We also disable the data terms for pixels that are marked as occluded in the occlusion mask, so that their flow value is chiefly determined by the epipolar and smoothness terms. For all terms, $\Psi\left(s^{2}\right)=\sqrt{s^{2}+10^{-6}}$ is the regularised $\ell_{1}$ penaliser. We use $\left(\alpha_{i}, \beta_{1}, \beta_{2}, \beta_{3}\right)=(10,31,60,200)$ for all results.

The second term of the energy favours correspondences that satisfy the epipolar constraint between $I_{1}$ and $I_{2}$ :

$$
\begin{aligned}
& E_{\mathrm{E}}^{1}=\Psi\left(\left(\left(\mathbf{x}+\mathbf{u}_{2}\right)^{\top} \mathbf{F}_{t} \mathbf{x}\right)^{2}\right), \\
& E_{\mathrm{E}}^{2}=\Psi\left(\left(\left(\mathbf{x}+\mathbf{u}_{1}+\mathbf{u}_{2}+\mathbf{u}_{3}\right)^{\top} \mathbf{F}_{t+1}\left(\mathbf{x}+\mathbf{u}_{1}\right)\right)^{2}\right),
\end{aligned}
$$

where $\mathbf{F}_{t}$ and $\mathbf{F}_{t+1}$ are the fundamental matrices at times $t$ and $t+1$. Note that the variational formulation uses different

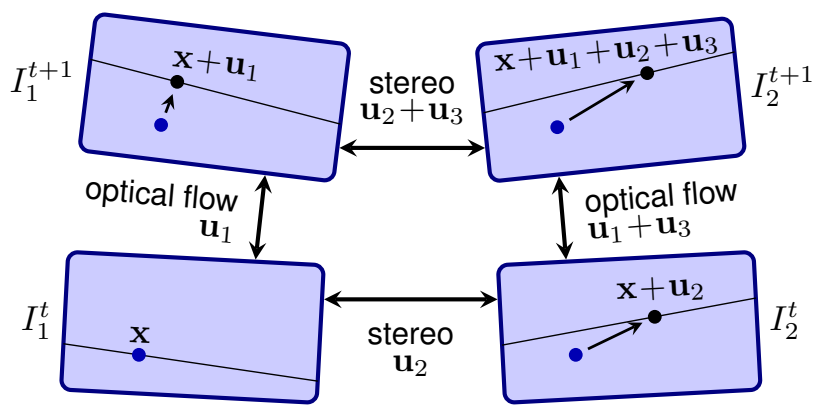

Figure 13. Four-frame configuration used in scene flow computation. data and epipolar terms than our matching cost (Equation 1), as the terms used in our variational formulation are sufficient when provided with a good initialisation, as in our case.

The last term imposes regularized total-variation smoothness - the standard TV norm is defined as $\|\nabla \mathbf{u}\|_{2}[40]$ - on the estimated flows by penalising their spatial derivatives:

$$
E_{\mathrm{S}}^{i}=\Psi\left(\left\|\nabla \mathbf{u}_{i}\right\|_{2}^{2}\right), \quad \text { for } i=1,2,3
$$

\section{B. Camera motion in the used datasets}

Most of the datasets we use in our paper (BEAR, BOAR, BOY, DEER) were captured with independently moving, handheld cameras. This is clearly visible when looking at the camera baselines and angles between cameras over time, which are shown in Figure 14. The camera baselines vary by more than 50 percent, and up to 250 percent (DEER), while the angle between cameras varies over a range of 4 degrees (BOAR) to 36 degrees (DEER). The ODZEMOK dataset has a constant camera baseline, but the angle between cameras varies between about 10 and 20 degrees. The TRAFFIC 2 dataset (now shown in Figure 14) uses a fixed stereo calibration with constant baseline and parallel cameras for all video frames.

\section{References}

[1] S. Avidan and A. Shashua. Trajectory triangulation: 3D reconstruction of moving points from a monocular image sequence. IEEE Transactions on Pattern Analysis and Machine Intelligence, 22(4):348-357, 2000.

[2] L. Ballan, G. J. Brostow, J. Puwein, and M. Pollefeys. Unstructured video-based rendering: interactive exploration of casually captured videos. ACM Transactions on Graphics, 29 (4):87:1-11, 2010.

[3] C. Barnes, E. Shechtman, D. B. Goldman, and A. Finkelstein. The generalized PatchMatch correspondence algorithm. In ECCV, 2010.

[4] T. Basha, S. Avidan, A. Hornung, and W. Matusik. Structure and motion from scene registration. In CVPR, 2012.

[5] T. Basha, Y. Moses, and N. Kiryati. Multi-view scene flow estimation: A view centered variational approach. International Journal of Computer Vision, 101(1):6-21, 2013.

[6] F. Besse, C. Rother, A. Fitzgibbon, and J. Kautz. PMBP: PatchMatch belief propagation for correspondence field estimation. International Journal of Computer Vision, 110(1): 2-13, 2014.

[7] D. J. Butler, J. Wulff, G. B. Stanley, and M. J. Black. A naturalistic open source movie for optical flow evaluation. In ECCV, 2012.

[8] J. Čech, J. Sanchez-Riera, and R. Horaud. Scene flow estimation by growing correspondence seeds. In CVPR, 2011.

[9] Y. Dai, H. Li, and M. He. A simple prior-free method for non-rigid structure-from-motion factorization. International Journal of Computer Vision, 107(2):101-122, 2014.

[10] F. Devernay, D. Mateus, and M. Guilbert. Multi-camera scene flow by tracking 3-D points and surfels. In CVPR, 2006. 

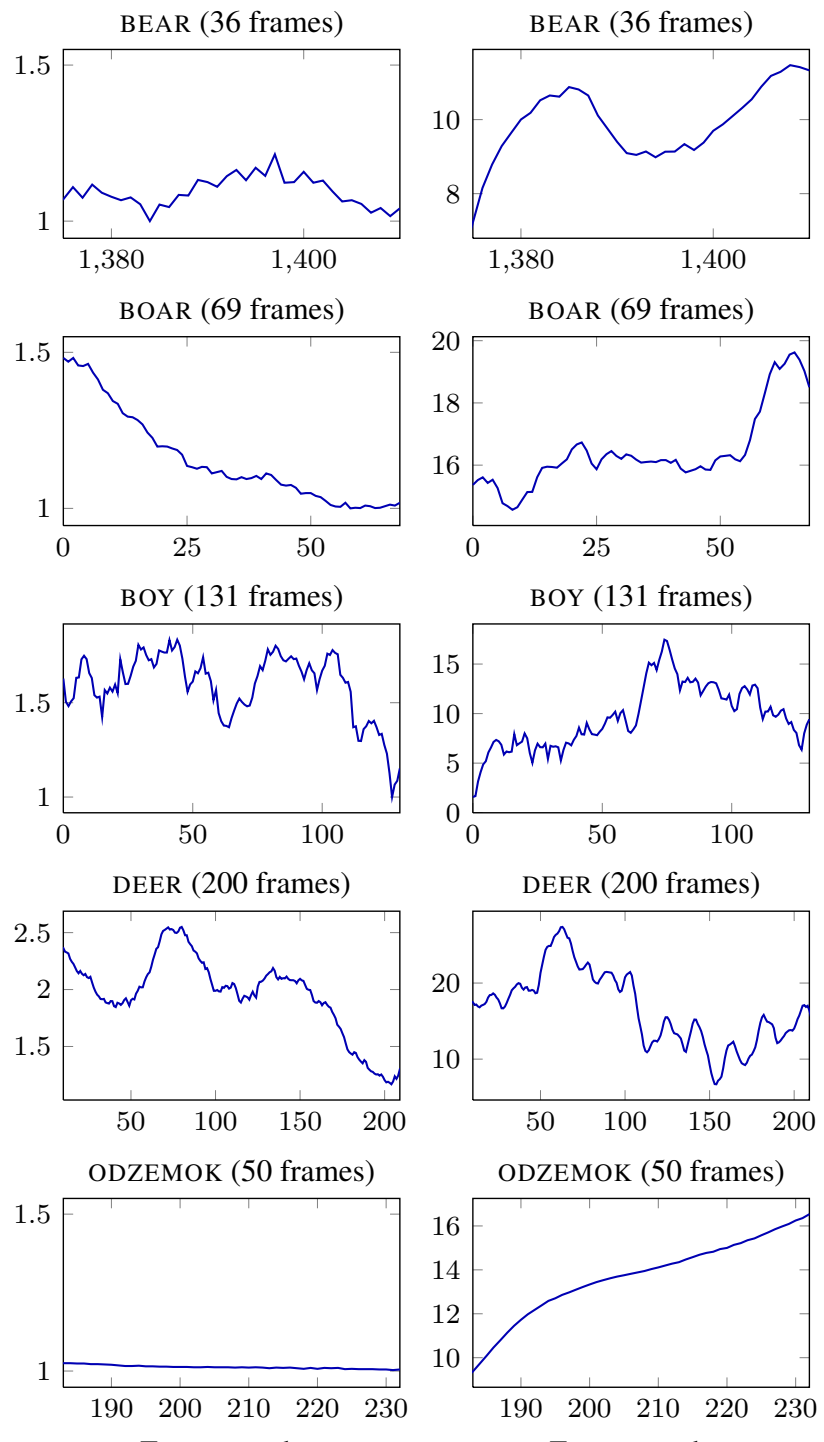

Frame number

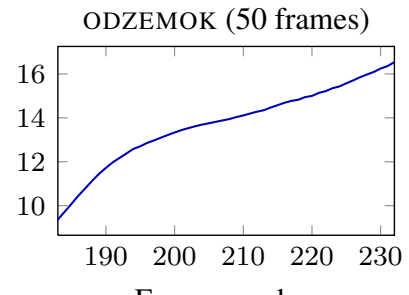

Figure 14. Visualisation of independent camera motion. Left: The baseline between cameras over time, normalised so that the minimum baseline is equal to one. Right: Angle between cameras over time (in degrees), specifically the angle between the principal axes of both cameras.

[11] A. Elhayek, C. Stoll, K. I. Kim, H.-P. Seidel, and C. Theobalt. Feature-based multi-video synchronization with subframe accuracy. In Pattern Recognition, 2012.

[12] D. Ferstl, G. Riegler, M. Rüther, and H. Bischof. CP-Census: A novel model for dense variational scene flow from RGB-D data. In $B M V C, 2014$.

[13] R. Garg, A. Roussos, and L. Agapito. Dense variational reconstruction of non-rigid surfaces from monocular video. In $C V P R, 2013$.

[14] T. Gaspar, P. Oliveira, and P. Favaro. Synchronization of two independently moving cameras without feature correspondences. In $E C C V, 2014$.
[15] S. Hadfield and R. Bowden. Scene particles: Unregularized particle-based scene flow estimation. IEEE Transactions on Pattern Analysis and Machine Intelligence, 36(3):564-576, 2014.

[16] R. Hartley and R. Vidal. Perspective nonrigid shape and motion recovery. In ECCV, 2008.

[17] R. Hartley and A. Zisserman. Multiple View Geometry in Computer Vision. Cambridge University Press, 2004.

[18] N. Hasler, B. Rosenhahn, T. Thormählen, M. Wand, J. Gall, and H.-P. Seidel. Markerless motion capture with unsynchronized moving cameras. In CVPR, 2009.

[19] E. Herbst, X. Ren, and D. Fox. RGB-D flow: Dense 3-D motion estimation using color and depth. In ICRA, 2013.

[20] M. Hornáček, A. Fitzgibbon, and C. Rother. SphereFlow: 6 DoF scene flow from RGB-D pairs. In CVPR, 2014.

[21] F. Huguet and F. Devernay. A variational method for scene flow estimation from stereo sequences. In ICCV, 2007.

[22] C. H. Hung, L. Xu, and J. Jia. Consistent binocular depth and scene flow with chained temporal profiles. International Journal of Computer Vision, 102(1-3):271-292, 2013.

[23] M. Jaimez, M. Souiai, J. Stückler, J. Gonzalez-Jimenez, and D. Cremers. Motion cooperation: Smooth piece-wise rigid scene flow from RGB-D images. In 3DV, 2015.

[24] H. Jiang, H. Liu, P. Tan, G. Zhang, and H. Bao. 3D reconstruction of dynamic scenes with multiple handheld cameras. In $E C C V, 2012$.

[25] J. Kannala and S. S. Brandt. Quasi-dense wide baseline matching using match propagation. In CVPR, 2007.

[26] A. Letouzey, B. Petit, and E. Boyer. Scene flow from depth and color images. In $B M V C, 2011$.

[27] A. Levin, D. Lischinski, and Y. Weiss. A closed-form solution to natural image matting. IEEE Transactions on Pattern Analysis and Machine Intelligence, 30(2):228-242, 2008.

[28] C. Lipski, F. Klose, and M. Magnor. Correspondence and depth-image based rendering a hybrid approach for freeviewpoint video. IEEE Transactions on Circuits and Systems for Video Technology, 24(6):942-951, 2014.

[29] M. A. Magnor, O. Grau, O. Sorkine-Hornung, and C. Theobalt, editors. Digital Representations of the Real World: How to Capture, Model, and Render Visual Reality. A K Peters, 2015.

[30] M. Menze and A. Geiger. Object scene flow for autonomous vehicles. In CVPR, 2015.

[31] B. Meyer, T. Stich, M. Magnor, and M. Pollefeys. Subframe temporal alignment of non-stationary cameras. In $B M V C$, 2008.

[32] D. Mishkin, J. Matas, M. Perdoch, and K. Lenc. WxBS: Wide baseline stereo generalizations. In BMVC, 2015.

[33] P. Moreels and P. Perona. Evaluation of features detectors and descriptors based on 3D objects. International Journal of Computer Vision, 73(3):263-284, 2007.

[34] A. Mustafa, H. Kim, J.-Y. Guillemaut, and A. Hilton. General dynamic scene reconstruction from multiple view video. In ICCV, 2015.

[35] H. S. Park, T. Shiratori, I. Matthews, and Y. Sheikh. 3D reconstruction of a moving point from a series of $2 \mathrm{D}$ projections. In $E C C V, 2010$. 
[36] J.-P. Pons, R. Keriven, and O. Faugeras. Multi-view stereo reconstruction and scene flow estimation with a global imagebased matching score. International Journal of Computer Vision, 72(2):179-193, 2007.

[37] J. Quiroga, T. Brox, F. Devernay, and J. Crowley. Dense semirigid scene flow estimation from RGBD images. In $E C C V$, 2014.

[38] J. Revaud, P. Weinzaepfel, Z. Harchaoui, and C. Schmid. EpicFlow: Edge-preserving interpolation of correspondences for optical flow. In $C V P R, 2015$.

[39] C. Richardt, D. Orr, I. Davies, A. Criminisi, and N. A. Dodgson. Real-time spatiotemporal stereo matching using the dual-cross-bilateral grid. In ECCV, 2010.

[40] L. I. Rudin, S. Osher, and E. Fatemi. Nonlinear total variation based noise removal algorithms. Physica D: Nonlinear Phenomena, 60(1-4):259-268, 1992.

[41] C. Russell, R. Yu, and L. Agapito. Video pop-up: Monocular 3D reconstruction of dynamic scenes. In ECCV, 2014.

[42] C. Strecha, T. Tuytelaars, and L. V. Gool. Dense matching of multiple wide-baseline views. In ICCV, 2003.

[43] D. Sun, E. B. Sudderth, and H. Pfister. Layered RGBD scene flow estimation. In CVPR, 2015.

[44] L. Thies, M. Zollhöfer, C. Richardt, C. Theobalt, and G. Greiner. Real-time halfway domain reconstruction of motion and geometry. In 3DV, 2016.

[45] E. Tola, V. Lepetit, and P. Fua. DAISY: An efficient dense descriptor applied to wide-baseline stereo. IEEE Transactions on Pattern Analysis and Machine Intelligence, 32(5):815-830, 2010.

[46] E. Tola, C. Strecha, and P. Fua. Efficient large-scale multiview stereo for ultra high-resolution image sets. Machine Vision and Applications, 23(5):903-920, 2012.

[47] T. Tuytelaars and K. Mikolajczyk. Local invariant feature detectors: A survey. Foundations and Trends in Computer Graphics and Vision, 3(3):177-280, 2008.

[48] L. Valgaerts, A. Bruhn, H. Zimmer, J. Weickert, C. Stoll, and C. Theobalt. Joint estimation of motion, structure and geometry from stereo sequences. In ECCV, 2010.

[49] L. Valgaerts, C. Wu, A. Bruhn, H.-P. Seidel, and C. Theobalt. Lightweight binocular facial performance capture under uncontrolled lighting. ACM Transactions on Graphics, 31(6): 187:1-11, 2012.

[50] S. Vedula, S. Baker, P. Rander, R. Collins, and T. Kanade. Three-dimensional scene flow. IEEE Transactions on Pattern Analysis and Machine Intelligence, 27(3):475-480, 2005.

[51] C. Vogel, S. Roth, and K. Schindler. View-consistent 3D scene flow estimation over multiple frames. In ECCV, 2014.

[52] C. Vogel, K. Schindler, and S. Roth. 3D scene flow estimation with a piecewise rigid scene model. International Journal of Computer Vision, 115(1):1-28, 2015.

[53] A. Wedel, T. Brox, T. Vaudrey, C. Rabe, U. Franke, and D. Cremers. Stereoscopic scene flow computation for 3D motion understanding. International Journal of Computer Vision, 95(1):29-51, 2011.

[54] J. Weickert. Anisotropic Diffusion in Image Processing. B.G. Teubner, 1998.
[55] C. Wu. VisualSFM: A visual structure from motion system, 2011. http://ccwu.me/vsfm/.

[56] C. Wu, C. Stoll, L. Valgaerts, and C. Theobalt. On-set performance capture of multiple actors with a stereo camera. ACM Transactions on Graphics, 32(6):161:1-11, 2013.

[57] R. Yu, C. Russell, N. D. F. Campbell, and L. Agapito. Direct, dense, and deformable: Template-based non-rigid 3D reconstruction from RGB video. In ICCV, 2015.

[58] A. Zanfir and C. Sminchisescu. Large displacement 3D scene flow with occlusion reasoning. In ICCV, 2015.

[59] L. Zhang, B. Curless, and S. M. Seitz. Spacetime stereo: shape recovery for dynamic scenes. In CVPR, 2003.

[60] Y. Zhang and C. Kambhamettu. On 3-D scene flow and structure recovery from multiview image sequences. IEEE Transactions on Systems, Man, and Cybernetics, 33(4):592606, 2003.

[61] E. Zheng, D. Ji, E. Dunn, and J.-M. Frahm. Sparse dynamic $3 \mathrm{D}$ reconstruction from unsynchronized videos. In $I C C V$, 2015.

[62] C. L. Zitnick, S. B. Kang, M. Uyttendaele, S. Winder, and R. Szeliski. High-quality video view interpolation using a layered representation. ACM Transactions on Graphics, 23 (3):600-608, 2004.

[63] D. Zou and P. Tan. CoSLAM: Collaborative visual SLAM in dynamic environments. IEEE Transactions on Pattern Analysis and Machine Intelligence, 35(2):354-366, 2013. 